\title{
Solvability of a Third-Order Multipoint Boundary Value Problem at Resonance
}

\author{
Zengji Du, ${ }^{1}$ Bensheng Zhao, ${ }^{1}$ and Zhanbing Bai $^{2}$ \\ ${ }^{1}$ School of Mathematics and Statistics Xuzhou, Jiangsu Normal University, Jiangsu 221116, China \\ ${ }^{2}$ School of Mathematics and Systems Science, Shandong University of Science and Technology, Qingdao 266510, China
}

Correspondence should be addressed to Zengji Du; duzengji@163.com

Received 10 December 2013; Accepted 13 January 2014; Published 23 February 2014

Academic Editor: Jifeng Chu

Copyright (c) 2014 Zengji Du et al. This is an open access article distributed under the Creative Commons Attribution License, which permits unrestricted use, distribution, and reproduction in any medium, provided the original work is properly cited.

We discuss a third-order multipoint boundary value problem under some appropriate resonance conditions. By using the coincidence degree theory, we establish the existence result of solutions. The emphasis here is that the dimension of the linear operator is equal to two. Our results supplement other results.

\section{Introduction}

In this paper, we are concerned with the following third-order ordinary differential equation:

$$
x^{\prime \prime \prime}(t)=f\left(t, x(t), x^{\prime}(t), x^{\prime \prime}(t)\right)+e(t), \quad t \in(0,1),
$$

with the boundary conditions

$$
x(0)=\sum_{i=1}^{m-2} \alpha_{i} x\left(\xi_{i}\right), \quad x^{\prime}(0)=0, \quad x(1)=\beta x(\eta),
$$

where $f:[0,1] \times R^{3} \rightarrow R$ is a Carathéodory function, $e \in$ $L^{1}[0,1], 0<\xi_{1}<\cdots<\xi_{m-2}<1, \alpha_{i} \in R, i=1, \ldots, m-2$, $\beta \geq 0$, and $\eta \in(0,1)$.

In recent years, many authors have paid much attention to the existence of solutions for multipoint boundary value problems at resonance: we refer the readers to see [1-11]. If the linear equation $L x=x^{\prime \prime \prime}(t)=0$ with boundary conditions (2) has nontrivial solutions, that is, $\operatorname{dim} \operatorname{Ker} L \geq 1$, the BVP (1)-(2) is called a resonance problem. In [5-11], the authors all discussed the case that $\operatorname{dim} \operatorname{Ker} L=1$. In $[2,3]$, the authors established the existence results for resonance boundary value problems with the case of $\operatorname{dim} \operatorname{Ker} L=2$. However, we will show that some conditions such as $\Lambda \neq 0$ assumed in $[2,3]$ are not necessary. We establish existence of some solutions for BVP (1)-(2) by using the coincidence degree theory of Mawhin [12] at resonance.
According to the constant $\beta$, the BVP (1)-(2) is divided into the following five resonance cases:

$$
\begin{aligned}
& \left(C_{1}\right) \quad 0<\beta<1,(1-\beta) \sum_{i=1}^{m-2} \alpha_{i} \xi_{i}^{2}=\left(1-\sum_{i=1}^{m-2} \alpha_{i}\right)\left(\beta \eta^{2}-1\right) ; \\
& \left(C_{2}\right) \quad \beta=1, \sum_{i=1}^{m-2} \alpha_{i} \xi_{i}^{2}=0, \sum_{i=1}^{m-2} \alpha_{i}=1 ; \\
& \left(C_{3}\right) \quad 1<\beta<1 / \eta^{2},(1-\beta) \sum_{i=1}^{m-2} \alpha_{i} \xi_{i}^{2}=\left(1-\sum_{i=1}^{m-2} \alpha_{i}\right)\left(\beta \eta^{2}-\right. \\
& 1) ; \\
& \left(C_{4}\right) \quad \beta=1 / \eta^{2}, \sum_{i=1}^{m-2} \alpha_{i} \xi_{i}^{2}=0, \sum_{i=1}^{m-2} \alpha_{i}=1 ; \\
& \left(C_{5}\right) \quad 1 / \eta^{2}<\beta,(1-\beta) \sum_{i=1}^{m-2} \alpha_{i} \xi_{i}^{2}=\left(1-\sum_{i=1}^{m-2} \alpha_{i}\right)\left(\beta \eta^{2}-1\right) .
\end{aligned}
$$

$\mathrm{Du}$ et al. [1] studied the existence results of BVP (1)(2) under the resonance conditions $\left(C_{2}\right)$ and $\left(C_{4}\right)$, that is, $\operatorname{dimKer} L=1$, but they did not discuss the other three cases. In this paper, under the resonance conditions, $\left(C_{1}\right),\left(C_{3}\right)$, or $\left(C_{5}\right)$, we could imply $\operatorname{dimKer} L=2$; thus we supplement the results in [1].

The layout of this paper is as follows. In Section 2, we briefly present some notations and an abstract existence result due to Mawhin. In Section 3, we study BVP (1)-(2) under the condition $\left(C_{1}\right)$ and obtain some existence results. In Section 4, we give an example of the existence results in Section 3.

\section{Preliminary}

Now, we briefly recall some notations and an abstract existence result by Mawhin [12]. 
Let $Y, Z$ be real Banach spaces and let $L: \operatorname{dom} L C$ $Y \rightarrow Z$ be a linear operator which is a Fredholm map of index zero and let $P: Y \rightarrow Y, Q: Z \rightarrow Z$ be continuous projectors such that $\operatorname{Im} P=\operatorname{Ker} L, \operatorname{Ker} Q=\operatorname{Im} L$ and $Y=$ Ker $L \oplus \operatorname{Ker} P, Z=\operatorname{Im} L \oplus \operatorname{Im} Q$. It follows that $\left.L\right|_{\operatorname{dom} L \cap \operatorname{Ker} P}$ : $\operatorname{dom} L \cap \operatorname{Ker} P \rightarrow \operatorname{Im} L$ is invertible; we denote the inverse of that map by $K_{P}$. Let $\Omega$ be an open bounded subset of $Y$ such that $\operatorname{dom} L \cap \Omega \neq \phi$; the map $N: Y \rightarrow Z$ is said to be $L$-compact on $\bar{\Omega}$ if the map $Q N(\bar{\Omega})$ is bounded and $K_{P}(I-Q) N: \bar{\Omega} \rightarrow Y$ is compact. For more details we refer the reader to the lecture of Mawhin [12].

The theorem we use in this paper is Theorem IV.13 of [12].

Theorem 1. Let L be a Fredholm map of index zero and let $N$ be L-compact on $\bar{\Omega}$. Assume that the following conditions are satisfied.

(i) $L x \neq N x$ for every $(x, \lambda) \in[(\operatorname{dom} L \backslash \operatorname{Ker} L) \cap \partial \Omega] \times$ $(0,1)$.

(ii) $N x \notin \operatorname{Im} L$ for every $x \in \operatorname{Ker} L \cap \partial \Omega$.

(iii) $\operatorname{deg}\left(\left.Q N\right|_{\operatorname{Ker} L}, \Omega \cap \operatorname{Ker} L, 0\right) \neq 0$, where $Q: Z \rightarrow Z$ is a continuous projector as above with $\operatorname{Im} L=\operatorname{Ker} Q$.

Then the abstract equation $L x=N x$ has at least one solution in $\operatorname{dom} L \cap \bar{\Omega}$.

In the following, we will use the classical spaces $C[0,1], C^{1}[0,1], C^{2}[0,1]$, and $L^{1}[0,1]$. For $x \in C^{2}[0,1]$, we use the norms $\|x\|_{\infty}=\max _{t \in[0,1]}|x(t)|$ and $\|x\|=$ $\max \left\{\|x\|_{\infty},\left\|x^{\prime}\right\|_{\infty},\left\|x^{\prime \prime}\right\|_{\infty}\right\}$, denote the norm in $L^{1}[0,1]$ by $\|\cdot\|_{1}$, and define the Sobolev space $W^{3,1}(0,1)$ as

$$
\begin{aligned}
& W^{3,1}(0,1)=\left\{x:[0,1] \longrightarrow R \mid x, x^{\prime}, x^{\prime \prime}\right. \text { are absolutely } \\
&\text { continuous on } \left.[0,1] \text { with } x^{\prime \prime \prime} \in L^{1}[0,1]\right\} .
\end{aligned}
$$

Let $Y=C^{2}[0,1], Z=L^{1}[0,1]$, and define the linear operator $L: \operatorname{dom} L \subset Y \rightarrow Z$ as $L x=x^{\prime \prime \prime}, x \in \operatorname{dom} L$, where

$$
\begin{aligned}
\operatorname{dom} L=\{ & x \in W^{3,1}(0,1): \\
& x \text { satisfies boundary conditions (2) }\} .
\end{aligned}
$$

We define $N: Y \rightarrow Z$ as

$$
N x=f\left(t, x(t), x^{\prime}(t), x^{\prime \prime}(t)\right), \quad t \in(0,1) .
$$

Then BVP (1)-(2) can be written as $L x=N x$.

\section{Existence Results}

Lemma 2. If the condition $\left(C_{1}\right)$ holds, then there exist $p \in$ $\{1,2, \ldots, m-2\}, q \in N^{+}, q \geq p+1$, such that

$$
\begin{aligned}
\Lambda(p, q)= & \sum_{i=1}^{m-2} \alpha_{i} \xi_{i}^{q+2}\left(1-\beta \eta^{p+2}\right) \\
& -\sum_{i=1}^{m-2} \alpha_{i} \xi_{i}^{p+2}\left(1-\beta \eta^{q+2}\right) \neq 0,
\end{aligned}
$$

where $N^{+}=\{1,2, \ldots\}$.

Proof. We prove that, for any $l \in N$, there exists $k_{l} \in\{l(m-$ $2)+1, \ldots,(l+1)(m-2)\}$, such that $\sum_{i=1}^{m-2} \alpha_{i} \xi_{i}^{k_{l}+2} \neq 0$.

If else, one has $\sum_{i=1}^{m-2} \alpha_{i} \xi_{i}^{k_{l}+2}=0$, any $k_{l} \in\{l(m-2)+$ $1, l(m-2)+2, \ldots,(l+1)(m-2)\}$; that is,

$$
\left(\begin{array}{ccc}
\xi_{1}^{l(m-2)+3} & \ldots & \xi_{m-2}^{l(m-2)+3} \\
\vdots & \ddots & \vdots \\
\xi_{1}^{(l+1)(m-2)+2} & \ldots & \xi_{m-2}^{(l+2)(m-2)+2}
\end{array}\right)\left(\begin{array}{c}
\alpha_{1} \\
\vdots \\
\alpha_{m-2}
\end{array}\right)=\left(\begin{array}{c}
0 \\
\vdots \\
0
\end{array}\right) .
$$

Since

$$
\begin{aligned}
& \left|\begin{array}{ccc}
\xi_{1}^{l(m-2)+3} & \cdots & \xi_{m-2}^{l(m-2)+3} \\
\vdots & \ddots & \vdots \\
\xi_{1}^{(l+1)(m-2)+2} & \cdots & \xi_{m-2}^{(l+1)(m-2)+2}
\end{array}\right| \\
& =\prod_{j=1}^{m-2} \xi_{j}^{l(m-2)+3}\left|\begin{array}{ccc}
1 & \cdots & 1 \\
\xi_{1} & \cdots & \xi_{m-2} \\
\vdots & \ddots & \vdots \\
\xi_{1}^{m-3} & \cdots & \xi_{m-2}^{m-3}
\end{array}\right| \\
& =\prod_{j=1}^{m-2} \xi_{j}^{l(m-2)+3} \prod_{1 \leq i<j \leq m-2}\left(\xi_{j}-\xi_{i}\right) \neq 0,
\end{aligned}
$$

thus $\alpha_{i}=0, i=1,2, \ldots, m-2$.

It is clear that

$$
\begin{aligned}
& (1-\beta) \sum_{i=1}^{m-2} \alpha_{i} \xi_{i}^{2}=0 \\
& \left(1-\sum_{i=1}^{m-2} \alpha_{i}\right)\left(\beta \eta^{2}-1\right)=\beta \eta^{2}-1<0,
\end{aligned}
$$

which is a contradiction to the condition $\left(C_{1}\right)$.

Set

$$
S=\left\{k_{l} \in N^{+}: 1-\beta \eta^{p+2}=\frac{\sum_{i=1}^{m-2} \alpha_{i}^{\xi_{i}^{p+2}}\left(1-\beta \eta^{k_{l}+2}\right)}{\sum_{i=1}^{m-2} \alpha_{i} \xi_{i}^{k_{l}+2}}\right\} .
$$

Then $S$ is a finite set.

If else, there exists a monotone sequence $\left\{k_{l_{r}}\right\}, r=$ $1,2, \ldots, k_{l_{r}}<k_{l_{r+1}}$, such that

$$
1-\beta \eta^{p+2}=\frac{\sum_{i=1}^{m-2} \alpha_{i} \xi_{i}^{p+2}\left(1-\beta \eta^{k_{l_{r}}+2}\right)}{\sum_{i=1}^{m-2} \alpha_{i} \xi_{i}^{k_{l_{r}}+2}} .
$$

From $0<\beta \eta^{p+2}<1$, we get

$$
\sum_{i=1}^{m-2} \alpha_{i} \xi_{i}^{p+2} \neq 0
$$

Thus

$$
1-\beta \eta^{p+2}=\lim _{k_{l_{r}} \rightarrow \infty} \frac{\sum_{i=1}^{m-2} \alpha_{i} \xi_{i}^{p+2}\left(1-\beta \eta^{k_{l_{r}}+2}\right)}{\sum_{i=1}^{m-2} \alpha_{i} \xi_{i}^{k_{l_{r}}+2}}=\infty .
$$

So it is a contradiction. Thus the Lemma is proved. 
Lemma 3. Let $\left(C_{1}\right)$ hold and $\Lambda(p, q) \neq 0$; then $L$ : $\operatorname{dom} L \subset$ $Y \rightarrow Z$ is a Fredholm map of index zero. Furthermore, the linear continuous projector operator $Q: Z \rightarrow Z$ can be defined by

$$
\mathrm{Q} y(t)=\left(T_{1} y\right) t^{p-1}+\left(T_{2} y\right) t^{q-1},
$$

where

$$
\begin{aligned}
& T_{1} y= \frac{p(p+1)(p+2)}{\Lambda(p, q)} \\
& \times\left[\left(\beta \eta^{q+2}-1\right) Q_{1} y+\sum_{i=1}^{m-2} \alpha_{i} \xi_{i}^{q+2} Q_{2} y\right], \\
& T_{2} y=-\frac{q(q+1)(q+2)}{\Lambda(p, q)} \\
& \times {\left[\left(\beta \eta^{p+2}-1\right) Q_{1} y+\sum_{i=1}^{m-2} \alpha_{i} \xi_{i}^{p+2} Q_{2} y\right], } \\
& Q_{1} y=\sum_{i=1}^{m-2} \alpha_{i} \int_{0}^{\xi_{i}} \int_{0}^{s} \int_{0}^{\tau} y(v) d v d \tau d s, \\
& Q_{2} y=\int_{0}^{1} \int_{0}^{s} \int_{0}^{\tau} y(v) d v d \tau d s \\
& \quad-\beta \int_{0}^{\eta} \int_{0}^{s} \int_{0}^{\tau} y(v) d v d \tau d s .
\end{aligned}
$$

And the linear operator $K_{P}: \operatorname{Im} L \rightarrow \operatorname{dom} L \cap \operatorname{Ker} P$ can be written by

$$
K_{P} y(t)=\int_{0}^{t} \int_{0}^{s} \int_{0}^{\tau} y(v) d v d \tau d s, \quad y \in \operatorname{Im} L .
$$

Furthermore

$$
\left\|K_{P} y\right\| \leq\|y\|_{1}, \quad y \in \operatorname{Im} L .
$$

Proof. It is clear that $\operatorname{Ker} L=\left\{x \in \operatorname{dom} L: x=a+c t^{2}, a=\right.$ $\left.\left(\left(\beta \eta^{2}-1\right) /(1-\beta)\right) c, c \in R\right\}$.

Now we show that

$$
\operatorname{Im} L=\left\{y \in Z: Q_{1} y=Q_{2} y=0\right\} .
$$

The equation

$$
x^{\prime \prime \prime}=y,
$$

has a solution $x(t)$ satisfying (2) if and only if

$$
Q_{1} y=Q_{2} y=0 .
$$

In fact, if (19) has a solution $x(t)$ such that (2), then from (19) we have

$$
x(t)=\frac{\beta \eta^{2}-1}{1-\beta} c+c t^{2}+\int_{0}^{t} \int_{0}^{s} \int_{0}^{\tau} y(v) d v d \tau d s .
$$

According to the condition $\left(C_{1}\right)$, we obtain

$$
Q_{1} y=Q_{2} y=0 .
$$

On the other hand, if (20) holds, let

$$
x(t)=\frac{\beta \eta^{2}-1}{1-\beta} c+c t^{2}+\int_{0}^{t} \int_{0}^{s} \int_{0}^{\tau} y(v) d v d \tau d s
$$

where $c$ is an arbitrary constant; then $x(t)$ is a solution of (19) and (2). Hence (18) holds.

Set

$$
\begin{aligned}
T_{1} y= & \frac{p(p+1)(p+2)}{\Lambda(p, q)} \\
& \times\left[\left(\beta \eta^{q+2}-1\right) Q_{1} y+\sum_{i=1}^{m-2} \alpha_{i} \xi_{i}^{q+2} Q_{2} y\right], \\
T_{2} y= & -\frac{q(q+1)(q+2)}{\Lambda(p, q)} \\
& \times\left[\left(\beta \eta^{p+2}-1\right) Q_{1} y+\sum_{i=1}^{m-2} \alpha_{i} \xi_{i}^{p+2} Q_{2} y\right] .
\end{aligned}
$$

Then we define

$$
Q y(t)=\left(T_{1} y\right) t^{p-1}+\left(T_{2} y\right) t^{q-1} .
$$

It is clear that $\operatorname{dim} \operatorname{Im} Q=2$.

Again from

$$
\begin{aligned}
& T_{1}\left(\left(T_{1} y\right) t^{p-1}\right)= \frac{p(p+1)(p+2)}{\Lambda(p, q)} \\
& \times\left[\left(\beta \eta^{q+2}-1\right) Q_{1}\left(\left(T_{1} y\right) t^{p-1}\right)\right. \\
&\left.+\sum_{i=1}^{m-2} \alpha_{i} \xi_{i}^{q+2} Q_{2}\left(\left(T_{1} y\right) t^{p-1}\right)\right] \\
&=\frac{1}{\Lambda(p, q)}\left[\left(\beta \eta^{q+2}-1\right) p(p+1)\right. \\
& \times(p+2) Q_{1}\left(t^{p-1}\right) \\
&+p(p+1)(p+2) \\
&\left.\times \sum_{i=1}^{m-2} \alpha_{i} \xi_{i}^{q+2} Q_{2}\left(t^{p-1}\right)\right]
\end{aligned}
$$

$$
\times\left(T_{1} y\right)=T_{1} y,
$$




$$
\begin{aligned}
T_{1}\left(\left(T_{2} y\right) t^{q-1}\right)=\frac{p(p+1)(p+2)}{\Lambda(p, q)} & +T_{2}\left(\left(T_{1} y\right) t^{p-1}+\left(T_{2} y\right) t^{q-1}\right) t^{q-1} \\
\times\left[\left(\beta \eta^{q+2}-1\right) Q_{1}\left(\left(T_{2} y\right) t^{q-1}\right)\right. & =T_{1}\left(\left(T_{1} y\right) t^{p-1}\right) t^{p-1}+T_{1}\left(\left(T_{2} y\right) t^{q-1}\right) t^{p-1} \\
& +T_{2}\left(\left(T_{1} y\right) t^{p-1}\right) t^{q-1}+T_{2}\left(\left(T_{2} y\right) t^{q-1}\right) t^{q-1} \\
& =\left(T_{1} y\right) t^{p-1}+\left(T_{2} y\right) t^{q-1} \\
& =Q y .
\end{aligned}
$$$$
\times(p+2) Q_{1}\left(t^{q-1}\right)
$$$$
+p(p+1)(p+2)
$$$$
\left.\times \sum_{i=1}^{m-2} \alpha_{i} \xi_{i}^{q+2} Q_{2}\left(t^{q-1}\right)\right]
$$$$
\times\left(T_{2} y\right)=0
$$$$
T_{2}\left(\left(T_{1} y\right) t^{p-1}\right)=-\frac{q(q+1)(q+2)}{\Lambda(p, q)}
$$$$
\times\left[\left(\beta \eta^{p+2}-1\right) Q_{1}\left(\left(T_{1} y\right) t^{p-1}\right)\right.
$$$$
\left.+\sum_{i=1}^{m-2} \alpha_{i} \xi_{i}^{p+2} Q_{2}\left(\left(T_{1} y\right) t^{p-1}\right)\right]
$$$$
=-\frac{q(q+1)(q+2)}{\Lambda(p, q)}
$$$$
\times\left[\left(\beta \eta^{p+2}-1\right) Q_{1}\left(t^{p-1}\right)\right.
$$$$
\left.+\sum_{i=1}^{m-2} \alpha_{i} \xi_{i}^{p+2} Q_{2}\left(t^{p-1}\right)\right]
$$$$
\times\left(T_{1} y\right)=0 \text {, }
$$$$
T_{2}\left(\left(T_{2} y\right) t^{q-1}\right)=-\frac{q(q+1)(q+2)}{\Lambda(p, q)}
$$$$
\times\left[\left(\beta \eta^{p+2}-1\right) Q_{1}\left(\left(T_{2} y\right) t^{q-1}\right)\right.
$$$$
\left.+\sum_{i=1}^{m-2} \alpha_{i} \xi_{i}^{p+2} Q_{2}\left(\left(T_{2} y\right) t^{q-1}\right)\right]
$$$$
=T_{2} y \text {. }
$$

Thus the operator $Q$ is a projector.

Now we show that $\operatorname{Ker} Q=\operatorname{Im} L$. If $y \in \operatorname{Ker} Q$, from $Q y=$ 0 , we have

$$
\begin{aligned}
& \sum_{i=1}^{m-2} \alpha_{i} \xi_{i}^{q+2} Q_{2} y+\left(\beta \eta^{q+2}-1\right) Q_{1} y=0 \\
& \sum_{i=1}^{m-2} \alpha_{i} \xi_{i}^{p+2} Q_{2} y+\left(\beta \eta^{p+2}-1\right) Q_{1} y=0
\end{aligned}
$$

Because of

$$
\left|\begin{array}{ll}
\sum_{i=1}^{m-2} \alpha_{i} \xi_{i}^{q+2} & \beta \eta^{q+2}-1 \\
\sum_{i=1}^{m-2} \alpha_{i} \xi_{i}^{p+2} & \beta \eta^{p+2}-1
\end{array}\right|=-\Lambda(p, q) \neq 0,
$$

$Q_{1} y=Q_{2} y=0$, which yields $y \in \operatorname{Im} L$. On the other hand, if $y \in \operatorname{Im} L$, from $Q_{1} y=Q_{2} y=0$ and the definition of $Q$, so $Q y=0$; thus $y \in \operatorname{Ker} Q$. Hence, $\operatorname{Ker} Q=\operatorname{Im} L$.

For $y \in Z$, from $y=(y-Q y)+Q y,(y-Q y) \in \operatorname{Ker} Q=$ $\operatorname{Im} L, Q y \in \operatorname{Im} Q$, we have $Z=\operatorname{Im} L+\operatorname{Im} Q$. And if $y \epsilon$ $\operatorname{Im} L \cap \operatorname{Im} Q$, from $y \in \operatorname{Im} Q$, there exist constants $a, b \in R$, such that $y(t)=a t^{p-1}+b t^{q-1}$.

From $y \in \operatorname{Im} L$, we obtain

$$
\begin{aligned}
& a q(q+1)(q+2) \\
& \quad \times \sum_{i=1}^{m-2} \alpha_{i} \xi_{i}^{p+2}+b p(p+1)(p+2) \sum_{i=1}^{m-2} \alpha_{i} \xi_{i}^{q+2}=0 \\
& a q(q+1)(q+2)\left(1-\beta \eta^{p+2}\right) \\
& +b p(p+1)(p+2)\left(1-\beta \eta^{q+2}\right)=0 .
\end{aligned}
$$

In view of

$$
\begin{aligned}
& \left|\begin{array}{ll}
q(q+1)(q+2) \sum_{i=1}^{m-2} \alpha_{i} \xi_{i}^{p+2} & p(p+1)(p+2) \sum_{i=1}^{m-2} \alpha_{i} \xi_{i}^{q+2} \\
q(q+1)(q+2)\left(1-\beta \eta^{p+2}\right) & p(p+1)(p+2)\left(1-\beta \eta^{q+2}\right)
\end{array}\right| \\
& \quad=-q(q+1)(q+2) p(p+1)(p+2) \Lambda(p, q) \\
& \quad \neq 0
\end{aligned}
$$

$$
\begin{aligned}
Q^{2} y & =Q\left(\left(T_{1} y\right) t^{p-1}+\left(T_{2} y\right) t^{q-1}\right) \\
& =T_{1}\left(\left(T_{1} y\right) t^{p-1}+\left(T_{2} y\right) t^{q-1}\right) t^{p-1}
\end{aligned}
$$

therefore (30) has a unique solution $a=b=0$, which implies $\operatorname{Im} L \cap \operatorname{Im} Q=\{0\}$. So we have $Z=\operatorname{Im} L \oplus \operatorname{Im} Q$. Since 
$\operatorname{imKer} L=\operatorname{dim} \operatorname{Im} Q=\operatorname{codim} \operatorname{Im} L=2$, thus $L$ is a Fredholm map of index zero.

Let $P: Y \rightarrow Y$ be defined by

$$
P x(t)=x(0)+\frac{1}{2} x^{\prime \prime}(0) t^{2}, \quad t \in[0,1] .
$$

Then, the generalized inverse $K_{P}: \operatorname{Im} L \rightarrow \operatorname{dom} L \cap \operatorname{Ker} P$ can be written by

$$
K_{P} y(t)=\int_{0}^{t} \int_{0}^{s} \int_{0}^{\tau} y(v) d v d \tau d s, \quad y \in \operatorname{Im} L .
$$

In fact, for $y \in \operatorname{Im} L$, we have

$$
\left(L K_{P}\right) y(t)=\left(K_{P} y\right)^{\prime \prime \prime}=y(t),
$$

and for $x \in \operatorname{dom} L \cap \operatorname{Ker} P$, we know

$$
\begin{aligned}
\left(K_{P} L\right) x(t) & =\left(K_{P}\right) x^{\prime \prime \prime}(t) \\
& =\int_{0}^{t} \int_{0}^{s} \int_{0}^{\tau} x^{\prime \prime \prime}(v) d v d \tau d s \\
& =x(t)-\left[x(0)+\frac{1}{2} x^{\prime \prime}(0) t^{2}\right] \\
& =x(t)-P x(t) .
\end{aligned}
$$

Taking note that $x \in \operatorname{dom} L \cap \operatorname{Ker} P, P x(t)=0$, thus $\left(K_{P} L\right) x(t)=x(t)$.

It is clear that $\left\|K_{P} y\right\| \leq\|y\|_{1}$.

Theorem 4. Let the condition $\left(C_{1}\right)$ hold and $\Lambda(p, q) \neq 0$. Assume the following.

$\left(H_{1}\right)$ There exist functions $\alpha, \beta, \gamma, \theta \in L^{1}[0,1]$, such that

$$
\begin{aligned}
& \left|f\left(t, x_{1}, x_{2}, x_{3}\right)+e(t)\right| \\
& \leq \alpha(t)\left|x_{1}\right|+\beta(t)\left|x_{2}\right|+\gamma(t)\left|x_{3}\right|+\theta(t), \\
& \|\alpha\|_{1}+\|\beta\|_{1}+\|\gamma\|_{1}<1,
\end{aligned}
$$

where $\forall\left(x_{1}, x_{2}, x_{3}\right) \in R^{3}, t \in[0,1]$.

$\left(H_{2}\right)$ There exists a constant $A>0$ such that for $x \in$ $\operatorname{dom} L$, if $|x(t)|>A$ or $\left|x^{\prime \prime}(t)\right|>A$ for all $t \in[0,1]$, then

$$
Q_{1} N(x(t)) \neq 0 \quad \text { or } \quad Q_{2} N(x(t)) \neq 0 .
$$

$\left(H_{3}\right)$ There exists a constant $B>0$ such that for $a, c \in$ $R$, if $|a|>B$ or $|c|>B$, then either

$$
Q_{1} N\left(a+c t^{2}\right)+Q_{2} N\left(a+c t^{2}\right)>0
$$

or

$$
Q_{1} N\left(a+c t^{2}\right)+Q_{2} N\left(a+c t^{2}\right)<0 .
$$

Then BVP (1)-(2) has at least one solution in $C^{2}[0,1]$.
Proof. We divide the proof into the following steps.

Step 1. The set $\Omega_{1}=\{x \in \operatorname{dom} L \backslash \operatorname{Ker} L: L x=$ $\lambda N x$ for some $\lambda \in[0,1]\}$ is bounded.

For $x \in \Omega_{1}$, since $L x=\lambda N x$, so $\lambda \neq 0, N x \in \operatorname{Im} L$; hence

$$
Q_{1} N(x(t))=0, \quad Q_{2} N(x(t))=0 .
$$

From $\left(H_{2}\right)$, there exist $t_{0}, t_{1} \in[0,1]$ such that $\left|x\left(t_{0}\right)\right| \leq A$, $\left|x^{\prime \prime}\left(t_{1}\right)\right| \leq A . x, x^{\prime}$, and $x^{\prime \prime}$ are absolutely continuous for all $t \in[0,1]$, and

$$
\begin{gathered}
x(t)=x\left(t_{0}\right)+\int_{t_{0}}^{t} x^{\prime}(s) d s, \quad x^{\prime}(t)=x^{\prime}(0)+\int_{0}^{t} x^{\prime \prime}(s) d s \\
x^{\prime \prime}(t)=x^{\prime \prime}\left(t_{1}\right)+\int_{t_{1}}^{t} x^{\prime \prime \prime}(s) d s,
\end{gathered}
$$

which imply

$$
\begin{gathered}
\|x\|_{\infty} \leq A+\left\|x^{\prime}\right\|_{\infty}, \quad\left\|x^{\prime}\right\|_{\infty} \leq A+\left\|x^{\prime \prime \prime}\right\|_{1}, \\
\left\|x^{\prime \prime}\right\|_{\infty} \leq A+\left\|x^{\prime \prime \prime}\right\|_{1} .
\end{gathered}
$$

From $\left(H_{1}\right)$, we obtain

$$
\begin{aligned}
\left\|x^{\prime \prime \prime}\right\|_{1}= & \|L x\|_{1} \leq\|N x\|_{1} \\
\leq & \|\alpha\|_{1}\|x\|_{\infty}+\|\beta\|_{1}\left\|x^{\prime}\right\|_{\infty} \\
& +\|\gamma\|_{1}\left\|x^{\prime \prime}\right\|_{\infty}+\|\theta\|_{1} \\
\leq & \left(\|\alpha\|_{1}+\|\beta\|_{1}+\|\gamma\|_{1}\right)\left\|x^{\prime \prime \prime}\right\|_{1} \\
& +A\left(2\|\alpha\|_{1}+\|\beta\|_{1}+\|\gamma\|_{1}\right)+\|\theta\|_{1}, \\
\left\|x^{\prime \prime \prime}\right\|_{1} \leq & \frac{1}{1-\left(\|\alpha\|_{1}+\|\beta\|_{1}+\|\gamma\|_{1}\right)} \\
\times & {\left[A\left(2\|\alpha\|_{1}+\|\beta\|_{1}+\|\gamma\|_{1}\right)+\|\theta\|_{1}\right] . }
\end{aligned}
$$

So there exists a constant $M_{1}>0$ such that $\|x\| \leq M_{1}$; that is, the set $\Omega_{1}$ is bounded.

Step 2. The set $\Omega_{2}=\{x \in \operatorname{Ker} L: N x \in \operatorname{Im} L\}$ is bounded.

For $x \in \Omega_{2}, x \in$ Ker $L$ implies that $x=a+c t^{2}, a=$ $\left(\left(\beta \eta^{2}-1\right) /(1-\beta)\right) c, t \in[0,1], c \in R$. From $Q N x=0$, we get $Q_{1} N\left(a+c t^{2}\right)=Q_{2} N\left(a+c t^{2}\right)=0$. From $\left(H_{3}\right)$, then $\|x\| \leq|a|+|c| \leq 2 B$; that is, the set $\Omega_{2}$ is bounded.

Step 3. The set $\Omega_{3}=\{x \in \operatorname{Ker} L: \lambda J x+(1-\lambda) Q N x=0, \lambda \in$ $[0,1]\}$ is bounded.

For any $a, c \in R$, we define the linear isomorphism $J$ : $\operatorname{Ker} L \rightarrow \operatorname{Im} Q$ by

$$
J\left(a+c t^{2}\right)=\frac{a^{\prime} t^{p-1}+b^{\prime} t^{q-1}}{\Lambda(p, q)}
$$


where

$$
\begin{aligned}
& a^{\prime}=p(p+1)(p+2)\left[\left(\beta \eta^{q+2}-1\right)|a|+\sum_{i=1}^{m-2} \alpha_{i} \xi_{i}^{q+2}|c|\right], \\
& b^{\prime}=-q(q+1)(q+2)\left[\left(\beta \eta^{p+2}-1\right)|a|+\sum_{i=1}^{m-2} \alpha_{i} \xi_{i}^{p+2}|c|\right] .
\end{aligned}
$$

Set

$$
\begin{aligned}
& \mu=\frac{p(p+1)(p+2)}{\Lambda(p, q)}\left(\beta \eta^{q+2}-1\right), \\
& \nu=\frac{p(p+1)(p+2)}{\Lambda(p, q)} \sum_{i=1}^{m-2} \alpha_{i} \xi_{i}^{q+2}, \\
& \rho=-\frac{q(q+1)(q+2)}{\Lambda(p, q)}\left(\beta \eta^{p+2}-1\right), \\
& \omega=-\frac{q(q+1)(q+2)}{\Lambda(p, q)} \sum_{i=1}^{m-2} \alpha_{i} \xi_{i}^{p+2} .
\end{aligned}
$$

For any $x(t)=a+c t^{2} \in \Omega_{3}$, we obtain

$$
\begin{aligned}
& \mu\left[\lambda|a|+(1-\lambda) Q_{1} N\left(a+c t^{2}\right)\right] \\
& \quad+\nu\left[\lambda|c|+(1-\lambda) Q_{2} N\left(a+c t^{2}\right)\right]=0, \\
& \rho\left[\lambda|a|+(1-\lambda) Q_{1} N\left(a+c t^{2}\right)\right] \\
& \quad+\omega\left[\lambda|c|+(1-\lambda) Q_{2} N\left(a+c t^{2}\right)\right]=0 .
\end{aligned}
$$

On account of

$$
\left|\begin{array}{ll}
\mu & \nu \\
\rho & \omega
\end{array}\right|=-\frac{p(p+1)(p+2) q(q+1)(q+2)}{\Lambda(p, q)}
$$$$
\neq 0 \text {, }
$$

therefore, we have

$$
\begin{aligned}
& \lambda|a|+(1-\lambda) Q_{1} N\left(a+c t^{2}\right)=0, \\
& \lambda|c|+(1-\lambda) Q_{2} N\left(a+c t^{2}\right)=0 .
\end{aligned}
$$

If $\lambda=1$, then $a=c=0$. If $\lambda \neq 1$ and $|a|>B$ or $|c|>B$, from the above equality and (38), one has

$$
\begin{aligned}
\lambda(|a|+|c|)= & -(1-\lambda) \\
& \times\left[Q_{1} N\left(a+c t^{2}\right)+Q_{2} N\left(a+c t^{2}\right)\right]<0,
\end{aligned}
$$

which contradicts $\lambda(|a|+|c|) \geq 0$; thus $\|x\| \leq|a|+|c| \leq 2 B$. So the set $\Omega_{3}$ is bounded.

Step 4. If (39) holds, similar to the above argument, we can prove that the set

$$
\Omega_{3}=\{x \in \operatorname{Ker} L:-\lambda J x+(1-\lambda) Q N x=0, \lambda \in[0,1]\}
$$

is bounded too, where $J$ is defined in (44).
Now, we will prove that all conditions of Theorem 1 are satisfied.

Let $\Omega$ be an open bounded subset of $Y$ such that $\mathrm{U}_{i=1}^{3} \bar{\Omega}_{i} \subset$ $\Omega$. By the Arzelá-Ascoli theorem, we can prove that $K_{P}(I-$ Q) $N: \bar{\Omega} \rightarrow Y$ is compact, so $N$ is $L$-compact on $\bar{\Omega}$.

Then by the above argument, we have

(i) $L x \neq N x$ for every $(x, \lambda) \in[(\operatorname{dom} L \backslash \operatorname{Ker} L) \cap \partial \Omega] \times$ $(0,1)$;

(ii) $N x \notin \operatorname{Im} L$ for every $x \in \operatorname{Ker} L \cap \partial \Omega$

(iii) let $H(x, \lambda)= \pm \lambda J x+(1-\lambda) Q N x$.

According to the above argument in Steps 3 and 4, we know $H(x, \lambda) \neq 0$ for every $x \in \partial \Omega \cap$ Ker $L$. Thus, by using the homotopy property of degree, we have

$$
\begin{aligned}
& \operatorname{deg}\left(\left.Q N\right|_{\operatorname{Ker} L}, \Omega \cap \operatorname{Ker} L, 0\right) \\
& =\operatorname{deg}(H(\cdot, 0), \Omega \cap \operatorname{Ker} L, 0) \\
& =\operatorname{deg}(H(\cdot, 1), \Omega \cap \operatorname{Ker} L, 0) \\
& =\operatorname{deg}( \pm J, \Omega \cap \operatorname{Ker} L, 0) \\
& = \pm 1 \\
& \neq 0 .
\end{aligned}
$$

Then by Theorem 1, $L x=N x$ has at least one solution in $\operatorname{dom} L \cap \bar{\Omega}$; that is, BVP (1)-(2) has at least one solution in $C^{2}[0,1]$.

\section{Example}

Example 1. We consider the following boundary value problem:

$$
\begin{aligned}
& x^{\prime \prime \prime}(t)=t^{2}+4+\frac{3}{8} x\left(1+\frac{1}{2} \sin x^{\prime}\right) \\
& +\frac{1}{4} t \cos \left(x^{\prime \prime}\right)^{2}, \quad t \in(0,1), \\
& x(0)=\frac{68}{59} x\left(\frac{1}{2}\right), \quad x^{\prime}(0)=0, \quad x(1)=\frac{1}{2} x\left(\frac{1}{3}\right) . \\
& \text { Let } \\
& \begin{array}{l}
f\left(t, x(t), x^{\prime}(t), x^{\prime \prime}(t)\right) \\
=4+\frac{3}{8} x\left(1+\frac{1}{2} \sin x^{\prime}\right)+\frac{1}{4} t \cos \left(x^{\prime \prime}\right)^{2}, \quad e(t)=t^{2}, \\
m=3, \quad \alpha_{1}=\frac{68}{59}, \quad \beta=\frac{1}{2}, \quad \xi_{1}=\frac{1}{2}, \quad \eta=\frac{1}{3} .
\end{array}
\end{aligned}
$$

Then the condition $\left(C_{1}\right)$ holds. 
From Lemma 2 , one has $\Lambda(1,2)=-697 / 9558 \neq 0$. By Lemma 3, we define

$$
\begin{gathered}
Q_{1} y=\frac{68}{59} \int_{0}^{1 / 2} \int_{0}^{s} \int_{0}^{\tau} y(v) d v d \tau d s, \\
Q_{2} y=\int_{0}^{1} \int_{0}^{s} \int_{0}^{\tau} y(v) d v d \tau d s \\
\quad-\frac{1}{2} \int_{0}^{1 / 3} \int_{0}^{s} \int_{0}^{\tau} y(v) d v d \tau d s, \\
T_{1} y=-\frac{57348}{697}\left(-\frac{1}{162} Q_{1} y+\frac{17}{236} Q_{2} y\right) \\
T_{2} y=\frac{229392}{697}\left(-\frac{53}{54} Q_{1} y+\frac{17}{118} Q_{2} y\right) \\
K_{P} y(t)=\int_{0}^{t} \int_{0}^{s} \int_{0}^{\tau} y(v) d v d \tau d s, \quad y \in \operatorname{Im} L . \\
Q y(t)=\left(T_{1} y\right) t^{1-1}+\left(T_{2} y\right) t^{2-1}=T_{1} y+\left(T_{2} y\right) t .
\end{gathered}
$$

Since $\left|f\left(t, x(t), x^{\prime}(t), x^{\prime \prime}(t)\right)+e(t)\right| \leq(9 / 16)|x(t)|+t^{2}+$ $(1 / 4) t+4$, then $\alpha(t)=9 / 16, \beta(t)=\gamma(t)=0, \theta(t)=t^{2}+$ $(1 / 4) t+4$.

If $x(t)>1=A,\left|x^{\prime \prime}(t)\right|>1=A$, and $B=1$, one has

$$
\begin{aligned}
Q_{1} N x & =\frac{68}{59} \int_{0}^{1 / 2} \int_{0}^{s} \int_{0}^{\tau}\left(v^{2}+4+\frac{3}{8} x\left(1+\frac{1}{2} \sin x^{\prime}\right)\right. \\
& \left.+\frac{1}{4} v \cos \left(x^{\prime \prime}\right)^{2}\right) d v d \tau d s \\
\geq & \frac{68}{59} \int_{0}^{1 / 2} \int_{0}^{s} \int_{0}^{\tau}\left(v^{2}+4+\frac{3}{16}-\frac{1}{4} v\right) d v d \tau d s \\
= & \frac{68}{59} \int_{0}^{1 / 2} \int_{0}^{s} \int_{0}^{\tau}\left[\left(v-\frac{1}{8}\right)^{2}+\frac{267}{64}\right] d v d \tau d s \\
> & 0, \\
Q_{2} N x= & \int_{0}^{1} \int_{0}^{s} \int_{0}^{\tau}\left(v^{2}+4+\frac{3}{8} x\left(1+\frac{1}{2} \sin x^{\prime}\right)\right. \\
\geq & \frac{1}{2} \int_{0}^{1 / 3} \int_{0}^{s} \int_{0}^{\tau}\left(v^{2}+4+\frac{3}{8} x\left(1+\frac{1}{2} \sin x^{\prime}\right)\right. \\
& -\frac{1}{2} \int_{0}^{1 / 3} \int_{0}^{s} \int_{0}^{\tau}\left(v^{2}+4+\frac{1}{4} x\left(1+\frac{1}{2} \cos \left(x^{\prime \prime}\right)^{2}\right) d v d \tau d s\right. \\
& \left.+x^{\prime \prime} x^{\prime}\right) d v d \tau d s
\end{aligned}
$$

$$
\begin{aligned}
\geq & \frac{1}{2} \int_{0}^{1 / 3} \int_{0}^{s} \int_{0}^{\tau}\left(v^{2}+4+\frac{3}{16}-\frac{1}{4} v\right) d v d \tau d s \\
= & \frac{1}{2} \int_{0}^{1 / 3} \int_{0}^{s} \int_{0}^{\tau}\left[\left(v-\frac{1}{8}\right)^{2}+\frac{267}{64}\right] d v d \tau d s \\
>0, & Q_{1} N\left(2+t^{2}\right)+Q_{2} N\left(2+t^{2}\right)>0 .
\end{aligned}
$$

Then BVP (53) satisfies Theorem 4. So it has at least one solution in $C^{2}[0,1]$.

Remark 2. By using a similar method as employed in the above proof, we could obtain some similar results under the condition $\left(C_{3}\right)$ or $\left(C_{5}\right)$, then we omit them.

\section{Conflict of Interests}

The authors declare that there is no conflict of interests regarding to the publication of this paper.

\section{Acknowledgments}

This paper is sponsored by the Natural Science Foundation of China (11071205, 11101349, 61201431), the Natural Science Foundation of Jiangsu Province, and PAPD of Jiangsu Higher Education Institutions.

\section{References}

[1] Z. Du, X. Lin, and W. Ge, "On a third-order multi-point boundary value problem at resonance," Journal of Mathematical Analysis and Applications, vol. 302, no. 1, pp. 217-229, 2005.

[2] N. Kosmatov, "A multi-point boundary value problem with two critical conditions," Nonlinear Analysis: Theory, Methods \& Applications, vol. 65, no. 3, pp. 622-633, 2006.

[3] B. Liu and Z. Zhao, "A note on multi-point boundary value problems," Nonlinear Analysis: Theory, Methods \& Applications, vol. 67, no. 9, pp. 2680-2689, 2007.

[4] X. Lin, Z. Du, and F. Meng, "A note on a third-order multipoint boundary value problem at resonance," Mathematische Nachrichten, vol. 284, no. 13, pp. 1690-1700, 2011.

[5] B. Liu, "Solvability of multi-point boundary value problem at resonance. IV," Applied Mathematics and Computation, vol. 143, no. 2-3, pp. 275-299, 2003.

[6] W. Ge, The Boundary Value Problems of Nonlinear Ordinary Differential Equations, Science Press, Beijing, China, 2007.

[7] Z. Du, "Solvability of functional differential equations with multi-point boundary value problems at resonance," Computers \& Mathematics with Applications, vol. 55, no. 11, pp. 2653-2661, 2008.

[8] S. Liang and L. Mu, "Multiplicity of positive solutions for singular three-point boundary value problems at resonance," Nonlinear Analysis:Theory, Methods \& Applications, vol. 71, no. 7-8, pp. 2497-2505, 2009.

[9] H. Lian, H. Pang, and W. Ge, "Solvability for second-order three-point boundary value problems at resonance on a halfline," Journal of Mathematical Analysis and Applications, vol. 337, no. 2, pp. 1171-1181, 2008. 
[10] L. Yang and C. Shen, "On the existence of positive solution for a kind of multi-point boundary value problem at resonance," Nonlinear Analysis: Theory, Methods \& Applications, vol. 72, no. 11, pp. 4211-4220, 2010.

[11] R. Ma and Y. Yang, "Existence result for a singular nonlinear boundary value problem at resonance," Nonlinear Analysis: Theory, Methods \& Applications, vol. 68, no. 3, pp. 671-680, 2008.

[12] J. Mawhin, Topological Degree Methods in Nonlinear Boundary Value Problems, vol. 40 of NSFCBMS Regional Conference Series in Mathematics, American Mathematical Society, Providence, RI, USA, 1979. 


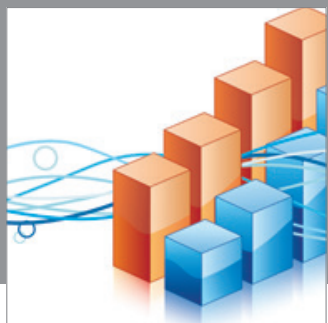

Advances in

Operations Research

mansans

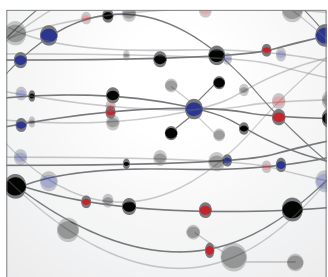

The Scientific World Journal
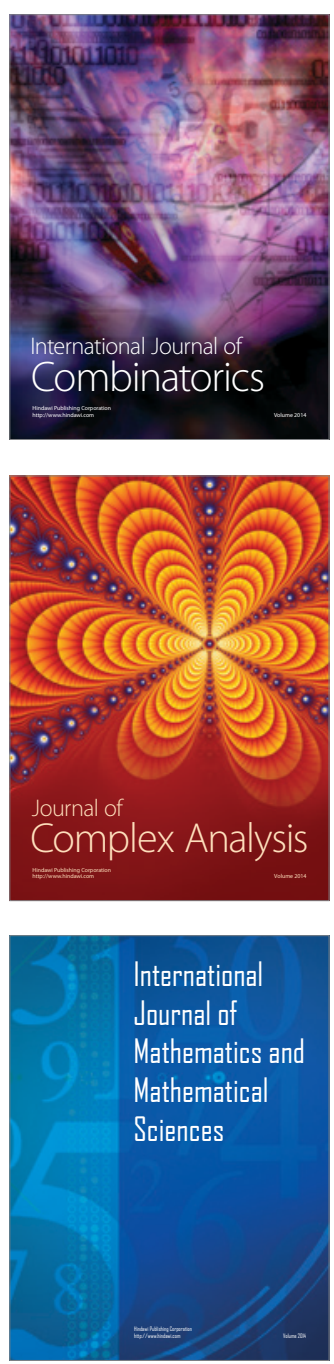
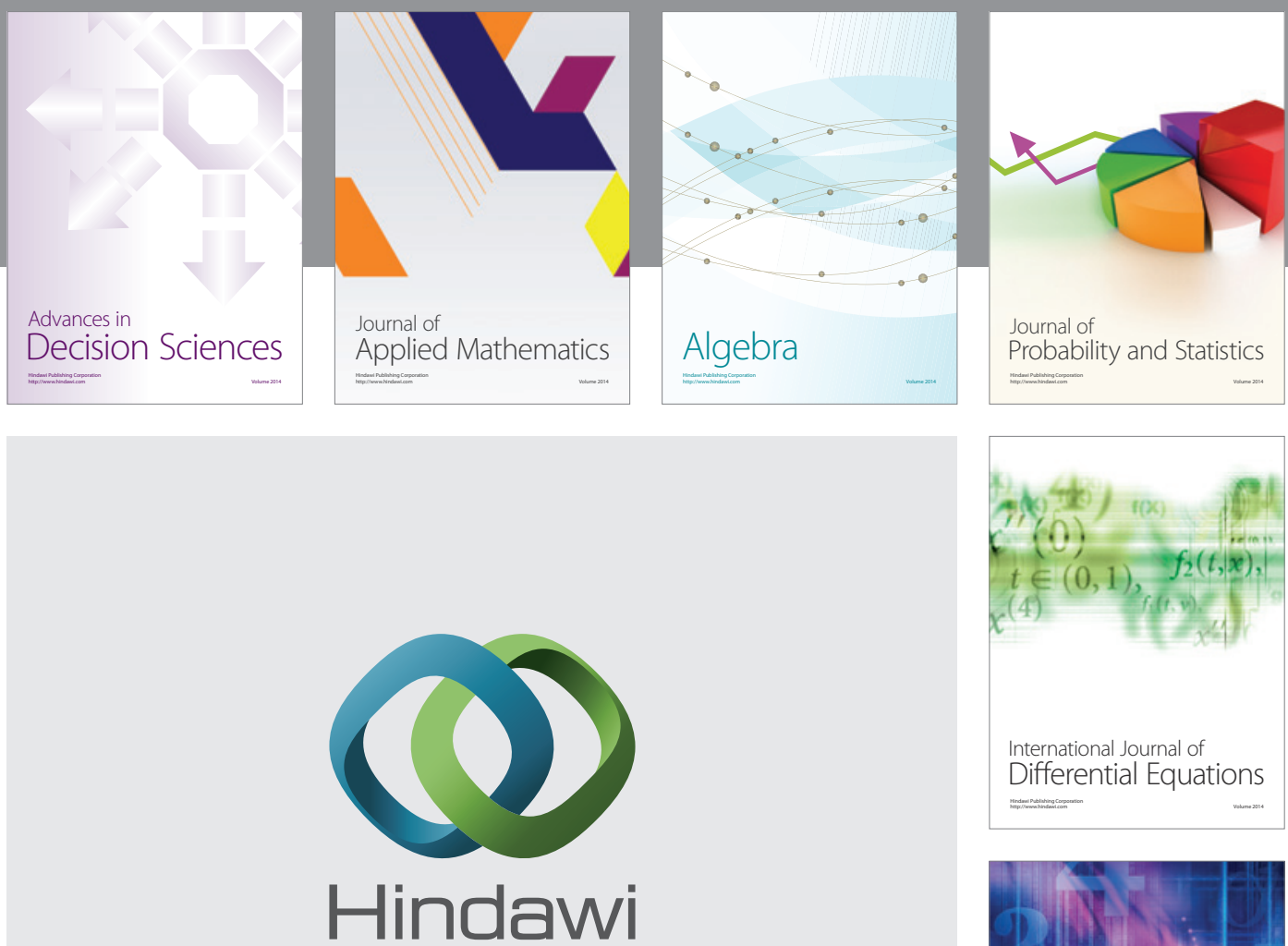

Submit your manuscripts at http://www.hindawi.com
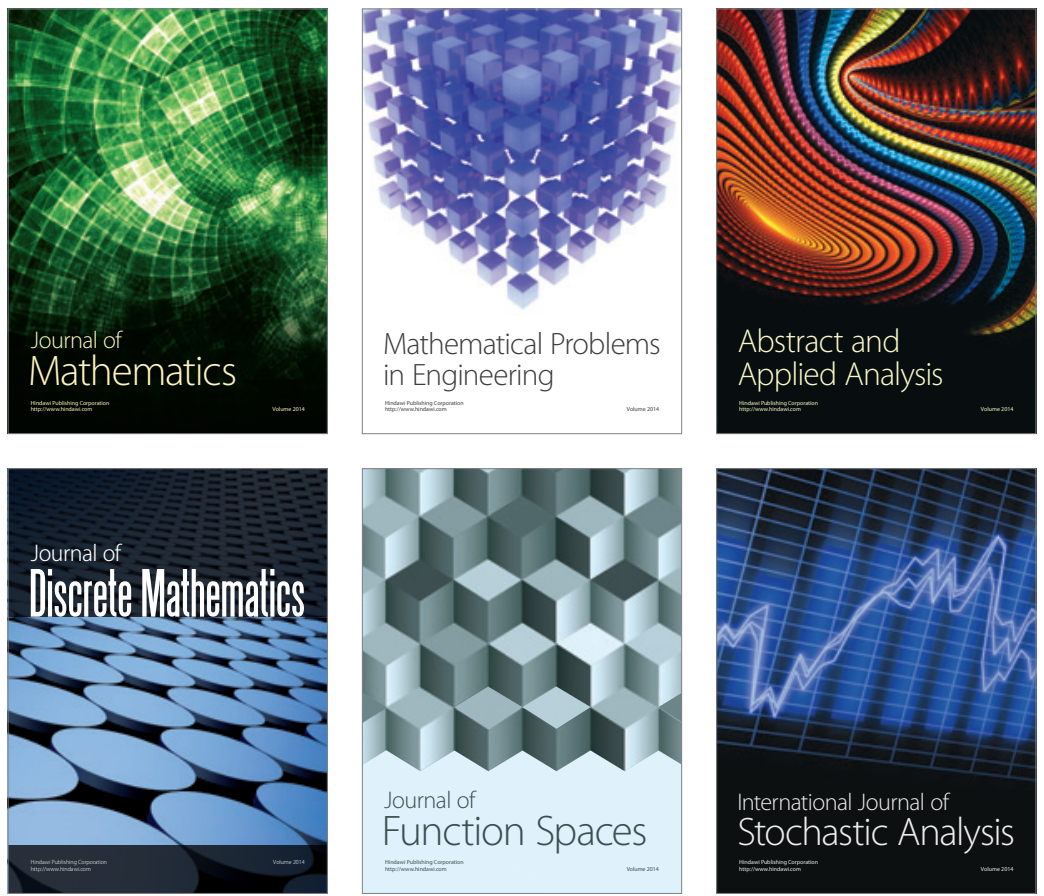

Journal of

Function Spaces

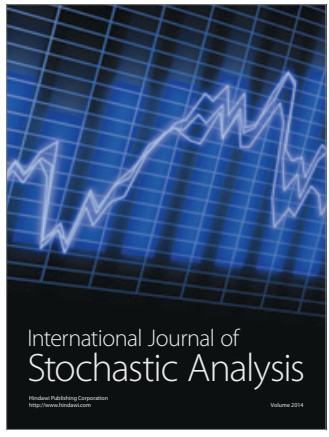

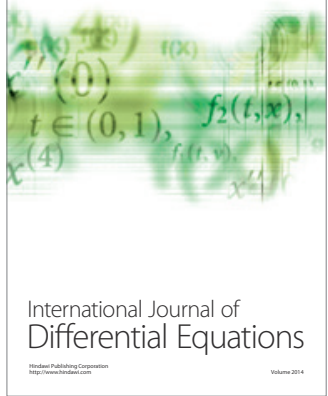
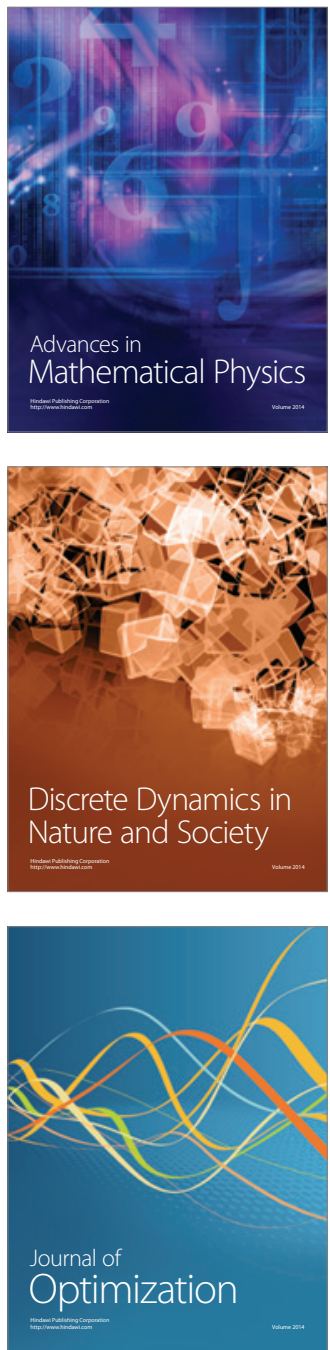